\title{
Local Fractional Derivative Boundary Value Problems for Tricomi Equation Arising in Fractal Transonic Flow
}

\author{
Xiao-Feng Niu, ${ }^{1}$ Cai-Li Zhang, ${ }^{1}$ Zheng-Biao $\mathrm{Li}^{2}{ }^{2}$ and Yang $\mathrm{Zhao}^{3}$ \\ ${ }^{1}$ College of Materials Science and Engineering, Taiyuan University of Technology, Taiyuan 030024, China \\ ${ }^{2}$ College of Mathematics and Information Science, Qujing Normal University, Qujing, Yunnan 655011, China \\ ${ }^{3}$ Department of Electronic and Information Technology, Jiangmen Polytechnic, Jiangmen 529090, China
}

Correspondence should be addressed to Yang Zhao; zhaoyang19781023@gmail.com

Received 19 June 2014; Accepted 26 June 2014; Published 13 July 2014

Academic Editor: Xiao-Jun Yang

Copyright (C) 2014 Xiao-Feng Niu et al. This is an open access article distributed under the Creative Commons Attribution License, which permits unrestricted use, distribution, and reproduction in any medium, provided the original work is properly cited. The local fractional decomposition method is applied to obtain the nondifferentiable numerical solutions for the local fractional
Tricomi equation arising in fractal transonic flow with the local fractional derivative boundary value conditions.

\section{Introduction}

The Tricomi equation [1] is the second-order linear partial differential equations of mixed type, which had been applied to describe the theory of plane transonic flow [2-7]. The Tricomi equation was used to describe the differentiable problems for the theory of plane transonic flow. However, for the fractal theory of plane transonic flow with nondifferentiable terms, the Tricomi equation is not applied to describe them. Recently, the local fractional calculus [8] was applied to describe the nondifferentiable problems, such as the fractal heat conduction $[8,9]$, the damped and dissipative wave equations in fractal strings [10], the local fractional Schrödinger equation [11], the wave equation on Cantor sets [12], the Navier-Stokes equations on Cantor sets [13], and others [14-19]. Recently, the local fractional Tricomi equation arising in fractal transonic flow was suggested in the form [19]

$$
\frac{y^{\alpha}}{\Gamma(1+\alpha)} \frac{\partial^{2 \alpha} u(x, y)}{\partial x^{2 \alpha}}+\frac{\partial^{2 \alpha} u(x, y)}{\partial y^{2 \alpha}}=0,
$$

where the quantity $u(x, y)$ is the nondifferentiable function, and the local fractional operator denotes [8]

$$
\frac{\partial^{\alpha} u(x, t)}{\partial t^{\alpha}}=\frac{\Delta^{\alpha}\left(u(x, t)-u\left(x, t_{0}\right)\right)}{\left(t-t_{0}\right)^{\alpha}},
$$

where

$$
\Delta^{\alpha}\left(u(x, t)-u\left(x, t_{0}\right)\right) \cong \Gamma(1+\alpha)\left[u(x, t)-u\left(x, t_{0}\right)\right] .
$$

The local fractional decomposition method [12] was used to solve the diffusion equation on Cantor time-space. The aim of this paper is to use the local fractional decomposition method to solve the local fractional Tricomi equation arising in fractal transonic flow with the local fractional derivative boundary value conditions. The structure of this paper is as follows. In Section 2, the local fractional integrals and derivatives are introduced. In Section 3, the local fractional decomposition method is suggested. In Section 4, the nondifferentiable numerical solutions for local fractional Tricomi equation with the local fractional derivative boundary value conditions are given. Finally, the conclusions are shown in Section 5.

\section{Local Fractional Integrals and Derivatives}

In this section, we introduce the basic theory of the local fractional integrals and derivatives [8-19], which are applied in the paper. 
Definition 1 (see [8-19]). For $\left|x-x_{0}\right|<\delta$, for $\varepsilon, \delta>0$ and $\varepsilon \in R$, we give the function $f(x) \in C_{\alpha}(a, b)$, when

$$
\left|f(x)-f\left(x_{0}\right)\right|<\varepsilon^{\alpha}, \quad 0<\alpha \leq 1,
$$

is valid.

Definition 2 (see [8-19]). Let $\left(t_{j}, t_{j+1}\right), j=0, \ldots, N-1, t_{0}=a$, and $t_{N}=b$ with $\Delta t_{j}=t_{j+1}-t_{j}$ and $\Delta t=\max \left\{\Delta t_{0}, \Delta t_{1}, \ldots\right\}$, be a partition of the interval $[a, b]$. The local fractional integral of $f(x)$ in the interval $[a, b]$ is defined as

$$
\begin{aligned}
{ }_{a} I_{b}^{(\alpha)} f(x) & =\frac{1}{\Gamma(1+\alpha)} \int_{a}^{b} f(t)(d t)^{\alpha} \\
& =\frac{1}{\Gamma(1+\alpha)} \lim _{\Delta t \rightarrow 0} \sum_{j=0}^{j=N-1} f\left(t_{j}\right)\left(\Delta t_{j}\right)^{\alpha} .
\end{aligned}
$$

As the inverse operator of (6), local fractional derivative of $f(x)$ of the order $\alpha$ in the interval $(a, b)$ is presented as [8-19]

$$
\frac{d^{\alpha} f\left(x_{0}\right)}{d x^{\alpha}}=D_{x}{ }^{(\alpha)} f\left(x_{0}\right)=\frac{\Delta^{\alpha}\left(f(x)-f\left(x_{0}\right)\right)}{\left(x-x_{0}\right)^{\alpha}},
$$

where

$$
\Delta^{\alpha}\left(f(x)-f\left(x_{0}\right)\right) \cong \Gamma(1+\alpha)\left[f(x)-f\left(x_{0}\right)\right] .
$$

The formulas of local fractional derivative and integral, which appear in the paper, are valid [8]:

$$
\begin{aligned}
\frac{d^{\alpha}}{d x^{\alpha}} \frac{x^{n \alpha}}{\Gamma(1+n \alpha)} & =\frac{x^{(n-1) \alpha}}{\Gamma(1+(n-1) \alpha)}, \quad n \in N, \\
\frac{d^{\alpha}}{d x^{\alpha}} E_{\alpha}\left(x^{\alpha}\right) & =E_{\alpha}\left(x^{\alpha}\right), \\
\frac{d^{\alpha}}{d x^{\alpha}} \sin _{\alpha}\left(x^{\alpha}\right) & =\cos _{\alpha}\left(x^{\alpha}\right), \\
\frac{d^{\alpha}}{d x^{\alpha}} \cos _{\alpha}\left(x^{\alpha}\right) & =-\sin _{\alpha}\left(x^{\alpha}\right), \\
{ }_{0} I_{x}^{(\alpha)} \frac{x^{(n-1) \alpha}}{\Gamma(1+(n-1) \alpha)} & =\frac{x^{n \alpha}}{\Gamma(1+n \alpha)}, \quad n \in N, \\
{ }_{0} I_{x}{ }^{(\alpha)} \cos _{\alpha}\left(x^{\alpha}\right) & =\sin _{\alpha}\left(x^{\alpha}\right) .
\end{aligned}
$$

\section{Analysis of the Method}

In this section, we give the local fractional decomposition method [12]. We consider the following local fractional operator equation in the form

$$
L_{\alpha}^{(2)} u+R_{\alpha} u=0
$$

where $L_{\alpha}^{(2)}$ is linear local fractional operators of the order $2 \alpha$ with respect to $x$ and $R_{\alpha}$ is the linear local fractional operators of order less than $2 \alpha$. We write (9) as

$$
L_{x x}^{(2 \alpha)} u+R_{\alpha} u=0
$$

where the $2 \alpha$-th local fractional differential operator denotes

$$
L_{\alpha}^{(n)}=L_{x x}^{(2 \alpha)}=\frac{\partial^{2 \alpha}}{\partial x^{2 \alpha}}
$$

and the linear local fractional operators of order less than $2 \alpha$ denote

$$
R_{\alpha}=\frac{\Gamma(1+\alpha)}{y^{\alpha}} \frac{\partial^{2 \alpha} u(x, y)}{\partial y^{2 \alpha}} .
$$

Define the $2 \alpha$-fold local fractional integral operator

$$
L_{\alpha}^{(-2 \alpha)} m(s)={ }_{0} I_{x}{ }^{(\alpha)}{ }_{0} I_{x}{ }^{(\alpha)} m(s)
$$

so that we obtain the local fractional iterative formula as follows:

$$
L_{\alpha}^{(-2 \alpha)} L_{x x}^{(2 \alpha)} u+L_{\alpha}^{(-2 \alpha)} L_{\alpha}^{(-2 \alpha)} R_{\alpha} u=0,
$$

which leads to

$$
u(x)=u_{0}(x)+L_{\alpha}^{(-2 \alpha)} L_{\alpha}^{(-2 \alpha)} R_{\alpha} u .
$$

Therefore, for $n \geq 0$, we obtain the recurrence formula in the form

$$
\begin{gathered}
u_{n+1}(x)=L_{\alpha}^{(-2)} R_{\alpha} u_{n}(x), \\
u_{0}(x)=r(x)
\end{gathered}
$$

Finally, the solution of (9) reads

$$
u(x)=\lim _{n \rightarrow \infty} \phi_{n}(x)=\lim _{n \rightarrow \infty} \sum_{n=0}^{\infty} u_{n}(x) .
$$

\section{The Nondifferentiable Numerical Solutions}

In this section, we discuss the nondifferentiable numerical solutions for the local fractional Tricomi equation arising in fractal transonic flow with the local fractional derivative boundary value conditions.

Example 1. We consider the initial-boundary value conditions for the local fractional Tricomi equation in the form [19]

$$
\begin{gathered}
u(0, y)=0, \\
u(l, y)=0, \\
u(x, 0)=\frac{x^{\alpha}}{\Gamma(1+\alpha)}, \\
\frac{\partial^{\alpha} u(x, 0)}{\partial x^{\alpha}}=\frac{x^{\alpha}}{\Gamma(1+\alpha)} .
\end{gathered}
$$

Using (20)-(21), we structure the recurrence formula in the form

$$
\begin{gathered}
u_{n+1}(x, y)=L_{\alpha}^{(-2)}\left[\frac{\Gamma(1+\alpha)}{y^{\alpha}} \frac{\partial^{2 \alpha} u_{n}(x, y)}{\partial y^{2 \alpha}}\right] \\
u_{0}(x, y)=\frac{x^{\alpha}}{\Gamma(1+\alpha)}+\frac{x^{\alpha}}{\Gamma(1+\alpha)} \frac{y^{\alpha}}{\Gamma(1+\alpha)}
\end{gathered}
$$


Hence, for $n=0$, the first term of (22) reads

$$
u_{1}(x, y)=0
$$

For $n=1$ the second term of (22) is given as

$$
u_{2}(x, y)=0
$$

Hence, we obtain

$$
u_{0}(x, y)=u_{1}(x, y)=\cdots=u_{n}(x, y)=0
$$

Finally, the solution of (9) with the local fractional derivative boundary value conditions (19)-(21) can be written as

$$
\begin{aligned}
u(x, y) & =\lim _{n \rightarrow \infty} \phi_{n}(x, y) \\
& =\lim _{n \rightarrow \infty} \sum_{n=0}^{\infty} u_{n}(x, y) \\
& =\frac{x^{\alpha}}{\Gamma(1+\alpha)}+\frac{x^{\alpha}}{\Gamma(1+\alpha)} \frac{y^{\alpha}}{\Gamma(1+\alpha)}
\end{aligned}
$$

which is in accordance with the result from the local fractional variational iteration method [19].

Example 2. Let us consider the initial-boundary value conditions for the local fractional Tricomi equation in the form

$$
\begin{gathered}
u(0, y)=0, \\
u(l, y)=0, \\
u(x, 0)=0, \\
\frac{\partial^{\alpha} u(x, 0)}{\partial x^{\alpha}}=\cos _{\alpha}\left(x^{\alpha}\right) .
\end{gathered}
$$

In view of (27), we set up the recurrence formula in the form

$$
\begin{gathered}
u_{n+1}(x, y)=L_{\alpha}^{(-2)}\left[\frac{\Gamma(1+\alpha)}{y^{\alpha}} \frac{\partial^{2 \alpha} u_{n}(x, y)}{\partial y^{2 \alpha}}\right], \\
u_{0}(x, y)=\cos _{\alpha}\left(x^{\alpha}\right) \frac{y^{\alpha}}{\Gamma(1+\alpha)} .
\end{gathered}
$$

Hence, from (28) we get the following equations:

$$
\begin{aligned}
u_{1}(x, y) & =L_{\alpha}^{(-2)}\left[\frac{\Gamma(1+\alpha)}{y^{\alpha}} \frac{\partial^{2 \alpha} u_{0}(x, y)}{\partial y^{2 \alpha}}\right] \\
& =L_{\alpha}^{(-2)}\left[\frac{\Gamma(1+\alpha)}{y^{\alpha}} \frac{\partial^{2 \alpha}}{\partial y^{2 \alpha}}\left(\cos _{\alpha}\left(x^{\alpha}\right) \frac{y^{\alpha}}{\Gamma(1+\alpha)}\right)\right] \\
& =0
\end{aligned}
$$

$$
\begin{aligned}
u_{2}(x, y) & =L_{\alpha}^{(-2)}\left[\frac{\Gamma(1+\alpha)}{y^{\alpha}} \frac{\partial^{2 \alpha} u_{1}(x, y)}{\partial y^{2 \alpha}}\right] \\
& =L_{\alpha}^{(-2)}\left[\frac{\Gamma(1+\alpha)}{y^{\alpha}} \frac{\partial^{2 \alpha}}{\partial y^{2 \alpha}}(0)\right] \\
& =0
\end{aligned}
$$

$$
\begin{aligned}
u_{3}(x, y) & =L_{\alpha}^{(-2)}\left[\frac{\Gamma(1+\alpha)}{y^{\alpha}} \frac{\partial^{2 \alpha} u_{2}(x, y)}{\partial y^{2 \alpha}}\right] \\
& =L_{\alpha}^{(-2)}\left[\frac{\Gamma(1+\alpha)}{y^{\alpha}} \frac{\partial^{2 \alpha}}{\partial y^{2 \alpha}}(0)\right] \\
& =0
\end{aligned}
$$$$
u_{4}(x, y)=L_{\alpha}^{(-2)}\left[\frac{\Gamma(1+\alpha)}{y^{\alpha}} \frac{\partial^{2 \alpha} u_{3}(x, y)}{\partial y^{2 \alpha}}\right]
$$$$
=L_{\alpha}^{(-2)}\left[\frac{\Gamma(1+\alpha)}{y^{\alpha}} \frac{\partial^{2 \alpha}}{\partial y^{2 \alpha}}(0)\right]
$$$$
=0 \text {, }
$$

Finally, we obtain the solution of (9) with the local fractional derivative boundary value conditions (27), namely,

$$
\begin{aligned}
u(x, y) & =\lim _{n \rightarrow \infty} \phi_{n}(x, y) \\
& =\lim _{n \rightarrow \infty} \sum_{n=0}^{\infty} u_{n}(x, y) \\
& =\cos _{\alpha}\left(x^{\alpha}\right) \frac{y^{\alpha}}{\Gamma(1+\alpha)},
\end{aligned}
$$

whose graph is shown in Figure 1.

Example 3. Let us consider the initial-boundary value conditions for the local fractional Tricomi equation in the form

$$
\begin{gathered}
u(0, y)=0, \\
u(l, y)=0, \\
u(x, 0)=\frac{x^{\alpha}}{\Gamma(1+\alpha)}, \\
\frac{\partial^{\alpha} u(x, 0)}{\partial x^{\alpha}}=\sin _{\alpha}\left(x^{\alpha}\right) .
\end{gathered}
$$




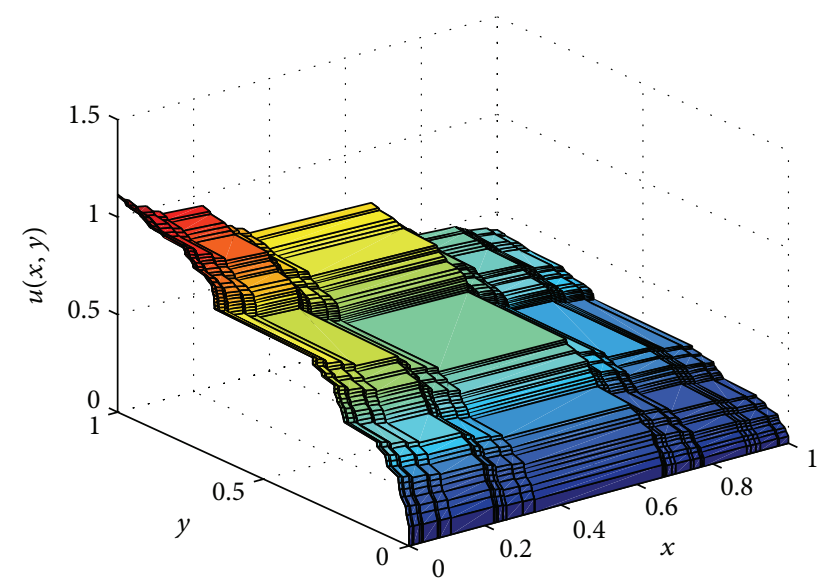

FIgURE 1: The plot of the solution of (9) with the local fractional derivative boundary value conditions (27) when $\alpha=\ln 2 / \ln 3$.

Making use of (31), the recurrence formula can be written as

$$
\begin{aligned}
& u_{n+1}(x, y)=L_{\alpha}^{(-2)}\left[\frac{\Gamma(1+\alpha)}{y^{\alpha}} \frac{\partial^{2 \alpha} u_{n}(x, y)}{\partial y^{2 \alpha}}\right] \\
& u_{0}(x, y)=\frac{x^{\alpha}}{\Gamma(1+\alpha)}+\sin _{\alpha}\left(x^{\alpha}\right) \frac{y^{\alpha}}{\Gamma(1+\alpha)}
\end{aligned}
$$

Appling (32) gives the following equations:

$$
\begin{aligned}
& u_{1}(x, y)=L_{\alpha}^{(-2)}\left[\frac{\Gamma(1+\alpha)}{y^{\alpha}} \frac{\partial^{2 \alpha} u_{0}(x, y)}{\partial y^{2 \alpha}}\right] \\
&=L_{\alpha}^{(-2)}\left[\frac{\Gamma(1+\alpha)}{y^{\alpha}} \frac{\partial^{2 \alpha}}{\partial y^{2 \alpha}}\right. \\
&\left.\times\left(\frac{x^{\alpha}}{\Gamma(1+\alpha)}+\sin _{\alpha}\left(x^{\alpha}\right) \frac{y^{\alpha}}{\Gamma(1+\alpha)}\right)\right] \\
&=0, \quad \\
& u_{2}(x, y)= L_{\alpha}^{(-2)}\left[\frac{\Gamma(1+\alpha)}{y^{\alpha}} \frac{\partial^{2 \alpha} u_{1}(x, y)}{\partial y^{2 \alpha}}\right] \\
&= L_{\alpha}^{(-2)}\left[\frac{\Gamma(1+\alpha)}{y^{\alpha}} \frac{\partial^{2 \alpha}}{\partial y^{2 \alpha}}(0)\right] \\
&= 0, \\
&= 0, \\
&= L_{\alpha}^{(-2)}\left[\frac{\Gamma(1+\alpha)}{y^{\alpha}} \frac{\partial^{2 \alpha}}{\partial y^{2 \alpha}}(0)\right] \\
& u_{3}(x, y) {\left[\frac{\Gamma(1+\alpha)}{y^{\alpha}} \frac{\partial^{2 \alpha} u_{2}(x, y)}{\partial y^{2 \alpha}}\right] }
\end{aligned}
$$

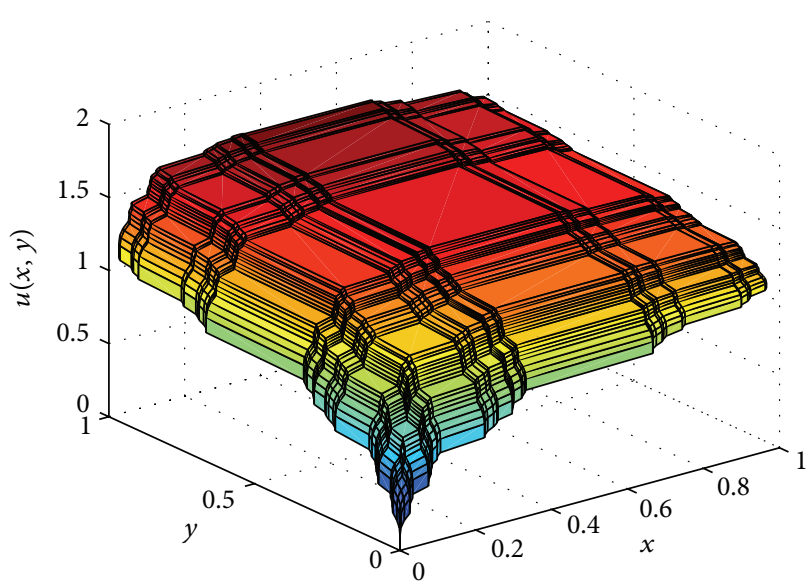

FIGURE 2: The plot of the solution of (9) with the local fractional derivative boundary value conditions (31) when $\alpha=\ln 2 / \ln 3$.

$$
\begin{aligned}
u_{4}(x, y) & =L_{\alpha}^{(-2)}\left[\frac{\Gamma(1+\alpha)}{y^{\alpha}} \frac{\partial^{2 \alpha} u_{3}(x, y)}{\partial y^{2 \alpha}}\right] \\
& =L_{\alpha}^{(-2)}\left[\frac{\Gamma(1+\alpha)}{y^{\alpha}} \frac{\partial^{2 \alpha}}{\partial y^{2 \alpha}}(0)\right] \\
& =0 \\
\vdots & \\
& u_{n}(x, y)=0 .
\end{aligned}
$$

Finally, the solution of (9) with the local fractional derivative boundary value conditions (31) reads

$$
\begin{aligned}
u(x, y) & =\lim _{n \rightarrow \infty} \phi_{n}(x, y) \\
& =\lim _{n \rightarrow \infty} \sum_{n=0}^{\infty} u_{n}(x, y) \\
& =\frac{x^{\alpha}}{\Gamma(1+\alpha)}+\sin _{\alpha}\left(x^{\alpha}\right) \frac{y^{\alpha}}{\Gamma(1+\alpha)},
\end{aligned}
$$

and its graph is shown in Figure 2.

\section{Conclusions}

In this work we discussed the nondifferentiable numerical solutions for the local fractional Tricomi equation arising in fractal transonic flow with the local fractional derivative boundary value conditions by using the local fractional decomposition method and their plots were also shown in the MatLab software.

\section{Conflict of Interests}

The authors declare that there is no conflict of interests regarding the publication of this paper. 


\section{Acknowledgment}

This work was supported by NSF110361048 of China and Yunnan province NSF Grant no. 2011FB090.

\section{References}

[1] F. Tricomi, On Second-Order Linear Partial Differential Equations of Mixed Type, Leningrad, Moscow, Russia, 1947.

[2] A. R. Manwell, "The Tricomi equation with applications to the theory of plane transonic flow," Recon Technical Report A 27617, 1979.

[3] D. Lupo and K. R. Payne, "A dual variational approach to a class of nonlocal semilinear Tricomi problems," Nonlinear Differential Equations and Applications, vol. 6, no. 3, pp. 247266, 1999.

[4] J. U. Kim, "An $L^{p}$ a priori estimate for the Tricomi equation in the upper half space," Transactions of the American Mathematical Society, vol. 351, no. 11, pp. 4611-4628, 1999.

[5] J. M. Rassias, "Uniqueness of quasi-regular solutions for a bi-parabolic elliptic bi-hyperbolic Tricomi problem," Complex Variables. Theory and Application, vol. 47, no. 8, pp. 707-718, 2002.

[6] K. Yagdjian, "Global existence for the $n$-dimensional semilinear Tricomi-type equations," Communications in Partial Differential Equations, vol. 31, no. 4-6, pp. 907-944, 2006.

[7] K. Yagdjian, "A note on the fundamental solution for the Tricomi-type equation in the hyperbolic domain," Journal of Differential Equations, vol. 206, no. 1, pp. 227-252, 2004.

[8] X.-J. Yang, Advanced Local Fractional Calculus and Its Applications, World Science, New York, NY, USA, 2012.

[9] A. M. Yang, Y. Z. Zhang, and Y. Long, "The Yang-Fourier transforms to heat-conduction in a semi-infinite fractal bar," Thermal Science, vol. 17, no. 3, pp. 707-713, 2013.

[10] W. H. Su, D. Baleanu, X. J. Yang et al., "Damped wave equation and dissipative wave equation in fractal strings within the local fractional variational iteration method," Fixed Point Theory and Applications, vol. 2013, no. 1, pp. 1-11, 2013.

[11] X. J. Yang, D. Baleanu, and J. A. T. Machado, "Mathematical aspects of Heisenberg uncertainty principle within local fractional Fourier analysis," Boundary Value Problems, no. 1, pp. 131146, 2013.

[12] D. Baleanu, J. A. Tenreiro Machado, C. Cattani, M. C. Baleanu, and X. Yang, "Local fractional variational iteration and decomposition methods for wave equation on Cantor sets within local fractional operators," Abstract and Applied Analysis, vol. 2014, Article ID 535048, 6 pages, 2014.

[13] X.-J. Yang, D. Baleanu, and J. T. Machado, "Systems of NavierStokes equations on Cantor sets," Mathematical Problems in Engineering, vol. 2013, Article ID 769724, 8 pages, 2013.

[14] G. Yi, "Local fractional Z transform in fractal space," Advances in Digital Multimedia, vol. 1, no. 2, pp. 96-102, 2012.

[15] X. Yang, H. M. Srivastava, J. H. He et al., "Cantor-type cylindrical-coordinate method for differential equations with local fractional derivatives," Physics Letters A, vol. 377, no. 2830, pp. 1696-1700, 2013.

[16] J.-H. He, "Asymptotic methods for solitary solutions and compactons," Abstract and Applied Analysis, vol. 2012, Article ID 916793, 130 pages, 2012.

[17] C.-G. Zhao, A.-M. Yang, H. Jafari, and A. Haghbin, “The YangLaplace transform for solving the IVPs with local fractional derivative," Abstract and Applied Analysis, vol. 2014, Article ID 386459, 5 pages, 2014.

[18] Z. Y. Chen, C. Cattani, and W. P. Zhong, "Signal processing for nondifferentiable data defined on Cantor sets: a local fractional Fourier series approach," Advances in Mathematical Physics, vol. 2014, Article ID 561434, 7 pages, 2014.

[19] A. M. Yang, Y. Z. Zhang, and X. L. Zhang, "The nondifferentiable solution for local fractional Tricomi equation arising in fractal transonic flow by loca 1 fractional variational iteration method," Advances in Mathematical Physics, vol. 2014, Article ID 983254, 6 pages, 2014. 


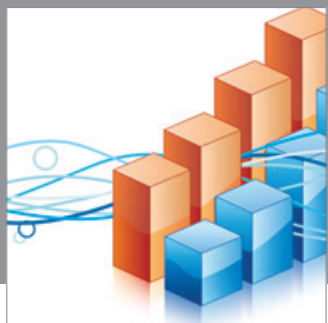

Advances in

Operations Research

mansans

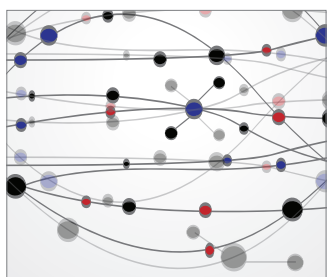

The Scientific World Journal
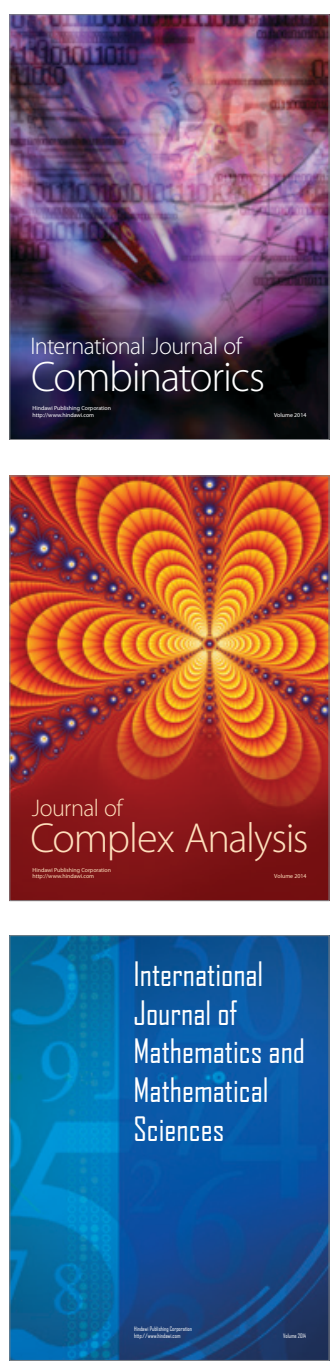
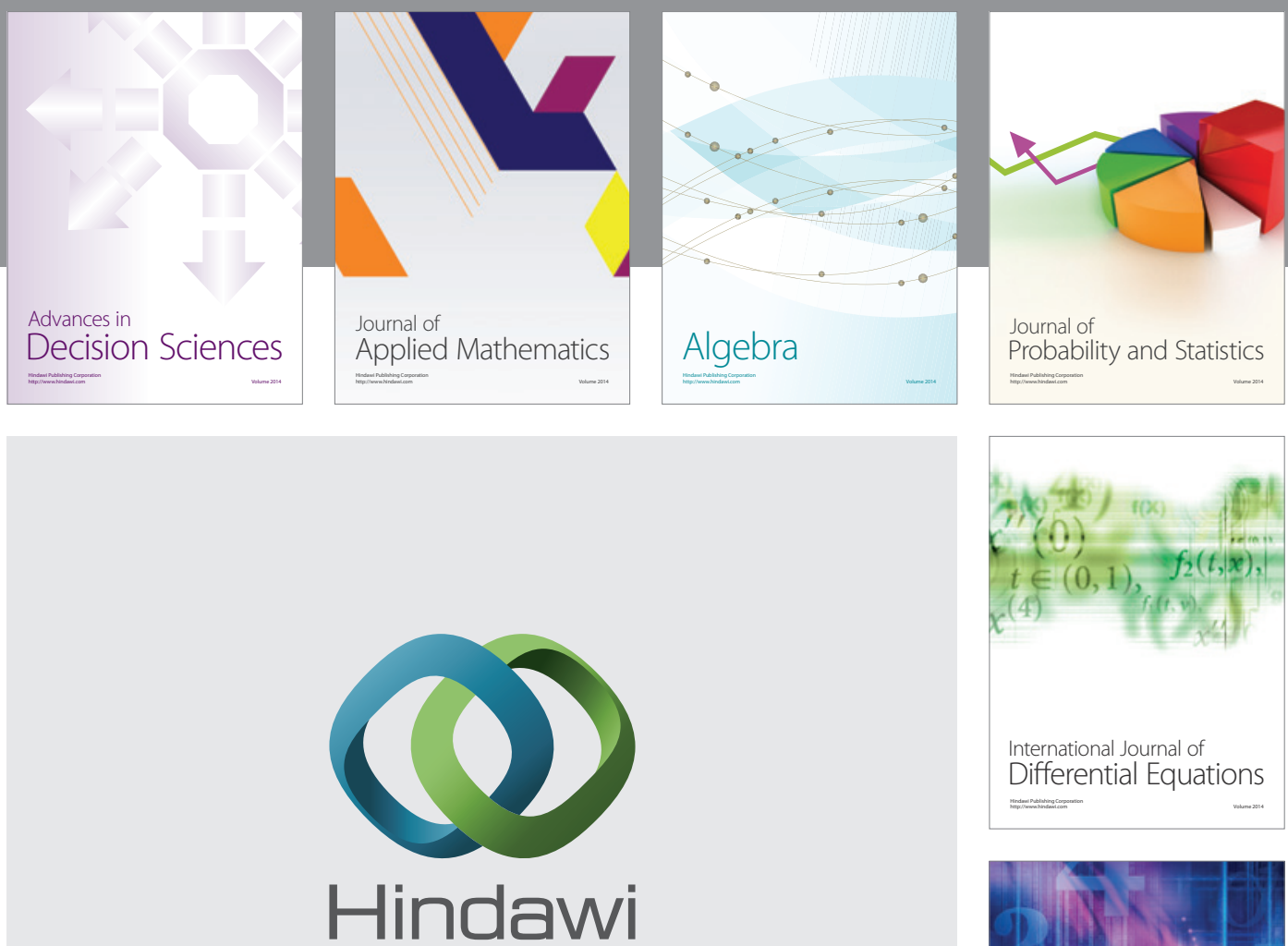

Submit your manuscripts at http://www.hindawi.com
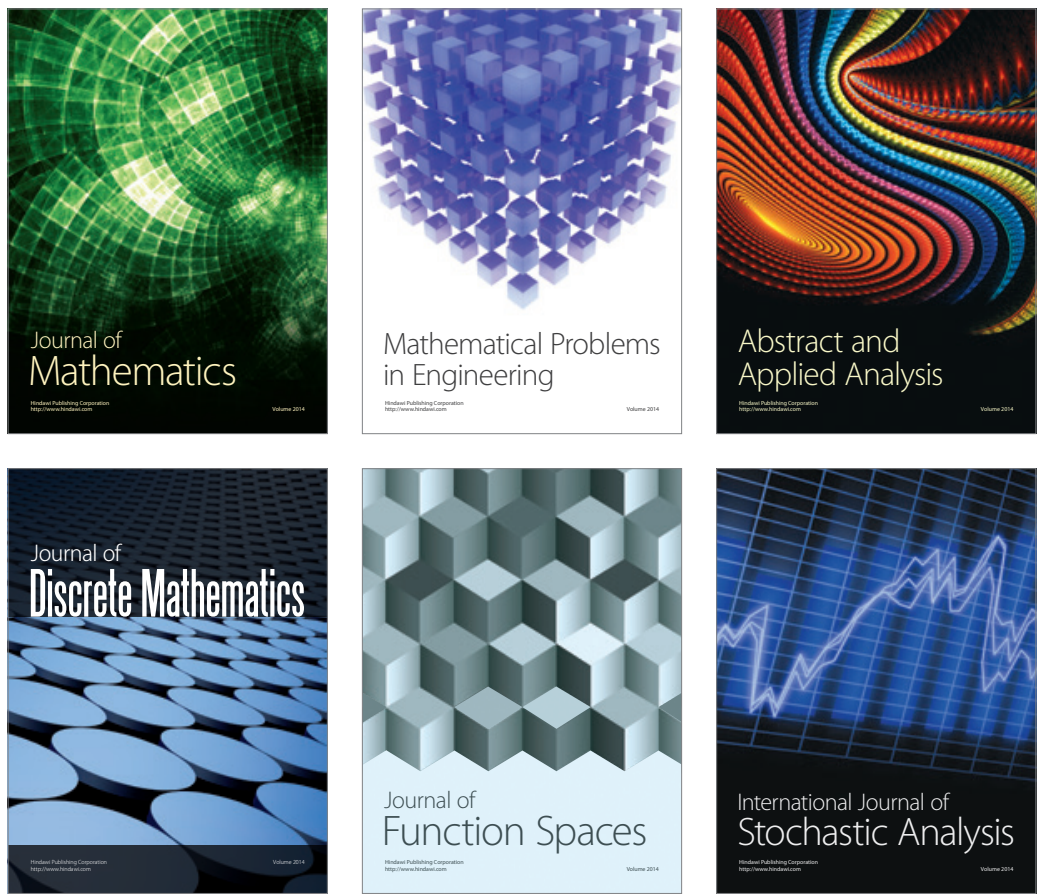

Journal of

Function Spaces

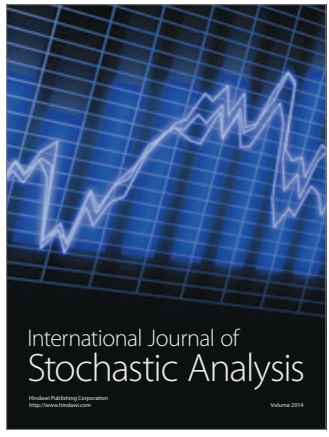

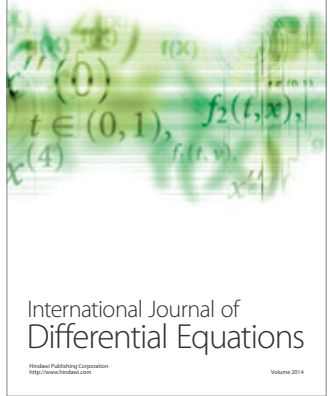
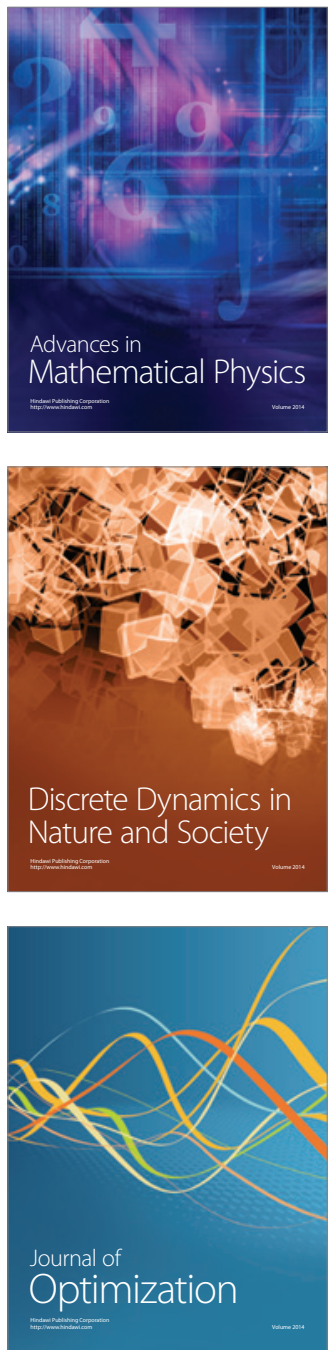